\title{
Knowledge and acceptability of prenatal diagnosis among pregnant women attending antenatal clinic in a tertiary health institution in Sokoto, Nigeria
}

\author{
Yakubu Ahmed ${ }^{1 *}$, Abubakar A. Panti ${ }^{1}$, Amina G. Umar ${ }^{1}$, Anas R. Funtua ${ }^{2}$, \\ Nafisa Abdullahi' ${ }^{3}$, Jamila A. Garba ${ }^{2}$
}

\begin{abstract}
${ }^{1}$ Department of Obstetrics and Gynecology, Usman Danfodiyo University, Sokoto, Nigeria
${ }^{2}$ Department of Obstetrics and Gynecology, ${ }^{3}$ Department of Medicine, Usman Danfodiyo University Teaching Hospital, Sokoto, Nigeria
\end{abstract}

Received: 19 June 2020

Revised: 07 August 2020

Accepted: 05 September 2020

\section{*Correspondence:}

Dr. Yakubu Ahmed,

E-mail: ahmeddryakubu32@gmail.com

Copyright: (C) the author(s), publisher and licensee Medip Academy. This is an open-access article distributed under the terms of the Creative Commons Attribution Non-Commercial License, which permits unrestricted non-commercial use, distribution, and reproduction in any medium, provided the original work is properly cited.

\begin{abstract}
Background: Technology and advances in research have made it possible for the fetus to become a patient whose illness can be investigated, diagnosed and treated in utero. The study was aimed at assessing the knowledge and acceptability of prenatal diagnosis among pregnant women.

Methods: This was a cross-sectional study carried out between December 2016 and March, 2017. Pregnant women attending antennal care clinic at the Usmanu Danfodiyo University Teaching Hospital, Sokoto (UDUTH) were recruited via convenient sampling method using semi-structured interviewer questionnaire.

Results: A total of 417 pregnant women were interviewed. The mean maternal age was $28.35 \pm 5$ years with a range between 17 to 45 years. Up to $188(69.10 \%)$ had at least secondary school level of education. There was an overall poor knowledge of prenatal diagnosis, as $406(97.36 \%)$ had little to no idea. There was statistically significant association between knowledge and educational status at $\mathrm{p}$ value- 0.0001 . Majority, $353(87.8 \%)$ will accept prenatal diagnosis if offered. There was also statistically significant association between educational status and acceptability as well as knowledge of prenatal diagnosis at $\mathrm{p}$ value- 0.001 and 0.001 respectively. The noninvasive method, ultrasound was the most preferred by the majority, $332(84.3 \%)$. Termination of pregnancy as an option of management in fetal abnormality was acceptable by up to $2 / 3(63.1 \%)$ of the respondents.

Conclusions: The knowledge of prenatal diagnosis among the respondents was generally poor. The identifiable factor that determined knowledge and acceptability of prenatal diagnosis was level of education.
\end{abstract}

Keywords: Acceptability, Knowledge, Management option, Prenatal diagnosis, Termination of pregnancy

\section{INTRODUCTION}

Prenatal diagnosis is the science of identifying malformations, disruptions, chromosomal abnormalities, and other genetic syndromes in the fetus. It encompasses routine screening tests for aneuploidy and neural-tube defects. ${ }^{1}$ Prenatal diagnosis first came into use in 1977 with the discovery of the significance of serum $\alpha$ fetoprotein (AFP). ${ }^{1}$ It is a well-known documented fact that congenital anomalies account for 20 to $25 \%$ of perinatal deaths, which is a major marker of quality of health care delivery. ${ }^{2}$

The goal of prenatal diagnosis is to provide accurate 
information regarding short- and long-term prognosis, recurrence risk, and potential therapy, and to improve counselling and outcomes. ${ }^{1}$ The objectives of prenatal diagnosis are; to give parents the chance to abort a fetus with a diagnosed condition to enable timely medical or surgical treatment of a condition before or after birth, and to give parent the chance to prepare psychologically, socially, financially and medically for a baby with a health problem or disability. ${ }^{3}$ The basic principles of practice in fetal medicine are based around the identification of highrisk pregnancies, accurate diagnosis of fetal anomalies using prenatal diagnostic techniques and a multidisciplinary approach to counselling of parents. ${ }^{4}$ This counselling will be centered on the accuracy of the diagnosis, prognosis for the fetus and neonate and the possibility of in utero therapy.

The indications for prenatal diagnosis include; maternal age above 35 years, family history of an inherited condition such as sickle cell disease, previous pregnancy with fetal abnormality, exposure to pathogens such as toxoplasmosis or rubella during pregnancy, exposure to teratogens such as, certain drugs or radiation while pregnant, women with type 1 diabetes mellitus, epilepsy or myotonic dystrophy among others. ${ }^{2}$

Screening tests assist in the selection of high risk patients on whom diagnostic test can be performed. Methods used include; maternal history, maternal biochemistry, maternal virology and ultrasound. ${ }^{5}$ However, when a screening test result suggests that the pregnancy is at high risk of an anomaly, then a definitive diagnostic test is offered to the child. However, some may be offered definitive diagnosis from the outset without any preceding screening tests.

The National Institute for Health and Clinical Excellence (NICE) and the UK National Screening Committee (UK NSC) have laid down standards for antenatal care, including the routine screening tests that should be offered to all pregnant women. ${ }^{6}$ However, these screening tests do not diagnose and may show the probability of a problem and in most cases, further diagnostic tests are required.

Maternal serum can be assayed for alpha-fetoprotein (AFP), a glycoprotein synthesized by the fetal yolk sac and later by the fetal gastrointestinal tract and liver. It is the major serum protein in the embryo and fetus and is thus analogous to albumin. Its concentration increases steadily in both fetal serum and amniotic fluid until 13 weeks' gestation, after which, levels rapidly decline. Conversely, AFP is found in steadily increasing quantities in maternal serum after 12 weeks. Defects in fetal integument, such as neural-tube and ventral wall defects, permit AFP to leak into the amniotic fluid, resulting in dramatically increased maternal serum levels. Maternal serum AFP screening is generally performed from 15 weeks through 20 weeks. $^{7}$ Several factors influence maternal serum AFP levels and are considered when calculating the AFP multiple of the median. ${ }^{6}$
Beta human chorionic gonadotrophin ( $\beta \mathrm{HCG})$ is used in conjunction with maternal serum AFP to screen for chromosomal abnormalities, Down's syndrome in particular where an elevated $\beta H C G$ coupled with decreased MSAFP suggestive. On the other hand, maternal estriol $\left(E_{3}\right)$, is dependent upon a viable fetus, a properly functioning placenta and maternal well-being. It tends to be lower when Down's syndrome is present and when there is adrenal hypoplasia with anencephaly. Additionally, inhibin A is secreted by the placenta and corpus luteum, and an increased level is associated with increased risk for trisomy 21. Accordingly, pregnancy associated plasma protein A (PAPP-A) level, when lower than normal value during first trimester may be associated with fetal chromosomal anomalies including trisomies. It can also predict adverse pregnancy outcome. ${ }^{7}$

Moreover, in the detection of certain trisomies, the triplemarker screening ( $\beta \mathrm{HCG}, \mathrm{E}_{3}$ and AFP) provides better sensitivity than any single marker alone. Nonetheless, the detection rate for trisomy 18 and trisomy 21 still remains quite low. ${ }^{7}$ According to the serum, urine and ultrasound screening study (SURUSS), integration of nuchal translucency measurement and pregnancy-associated plasma protein-A (PAPP-A) in the first trimester improves screening. This information in conjunction with early second-trimester measurement of AFP, $\mathrm{E}_{3}$, free $\beta$-hCG (or total hCG), and inhibin-A with maternal age provides the most effective method for screening of Down syndrome, with an $85 \%$ detection rate and $0.9 \%$ false-positive rate. ${ }^{7}$

For fetal component analysis, cytogenetic studies will include; amniocentesis that involve transabdominal aspiration of amniotic fluid, and that remains the most common procedure used in diagnosing fetal aneuploidy and other genetic conditions. ${ }^{1}$ It is usually performed between 15 weeks and 20 weeks' gestation but may be performed later. It is usually indicated to assess fetal karyotype, although use of FISH and array-based comparative genomic hybridization studies have increased considerably. The obtained amniocytes must be cultured before fetal karyotype can be assessed, therefore, the time needed for karyotyping is 7 to 10 days. ${ }^{1}$ Chorionic villus sampling (CVS) can be performed by biopsy of chorionic villi between 10 weeks and 13 weeks' gestation. ${ }^{1}$ Although most procedures are performed to assess fetal karyotype, numerous specialized genetic tests can also be performed. ${ }^{1}$ However, very few analyses specifically require either amniotic fluid or placental tissue. The primary advantage of villus biopsy is that results are available earlier in pregnancy, as full karyotype is available within 7 to 10 days, and some provide preliminary results within 48 hours allowing safer pregnancy termination, if desired. ${ }^{1,8}$

Fetal blood sampling in the form of cordocentesis or percutaneous umbilical blood sampling (PUBS) was initially described for fetal transfusion of red blood cells in cases of anemia from allo-immunization, and fetal anemia assessment remains the most common indication. Fetal blood sampling is also performed for assessment and 
treatment of platelet allo-immunization and for fetal karyotype determination, particularly in cases of mosaicism identified following amniocentesis or CVS. Fetal blood karyotyping can be accomplished within 24 to 48 hours. Thus, it is significantly quicker than the 7- to 10day turn-over time with amniocentesis or CVS. Although, fetal blood can be analysed for virtually any test performed on neonatal blood, improvements in tests available with amniocentesis and CVS have eliminated the need for fetal venipuncture in most cases..$^{1,8,9}$

Ultrasound screening for fetal anomalies varies considerably depending on the anomaly being screened for, gestational age, the skill of the operator and the quality of the equipment. ${ }^{4}$ The percentage of fetal anomalies detected by routine second trimester ultrasound screening according to anatomical systems include; central nervous system (76\%), urinary tract $(67 \%)$, pulmonary $(50 \%)$, gastrointestinal (42\%), skeletal (24\%) and cardiac $(17 \%){ }^{10}$ Integrated test which involve a nuchal translucency scan are likely to increase the detection of aneuploidy, major structural anomalies and cardiac defects earlier in gestation. A systematic review of routine ultrasound screening for fetal anomalies included 96,633 babies with detection rate of $44.7 \%$ which is higher among those $<24$ weeks of gestation $(41.3 \%)$ than $>24$ weeks $(18.6 \%) .{ }^{11}$ Soft-tissue markers are signs that can be detected on a second trimester anomaly scan that in themselves are not structural defects but have an association with aneuploidy, and therefore their presence increases the risk of aneuploidy. These markers include; nuchal skin edema, short femoral or humeral length measurements, choroid plexus cysts, bilateral renal pelvic dilatation, echogenic fetal bowel and hyper-echogenic foci ('golf balls') in the fetal heart. ${ }^{10,12}$

Following a diagnosis of fetal abnormality, the woman should be referred for appropriate counselling regarding the nature of the abnormality, the possibility of therapy, and the probable outcome for the child. It should also address the certainty of the diagnosis, the possible association with other anomalies and the associated risk of aneuploidy or other serious undiagnosed genetic syndromes. ${ }^{4}$ They also need to be advised on the prognosis for the fetus including perinatal morbidity including the risk of intrauterine death, the post-natal morbidity associated with the findings and the life expectancy for the child. Finally, they need to be counselled of any procedure to be offered while the fetus is in-utero, whether any curative or ameliorating procedures can be offered in the neonatal period, in addition to timing and mode of delivery. For chromosomal anomalies and many structural abnormalities not compatible with life, the management options are essentially limited to termination versus continuation of pregnancy. Unless the abnormality is trivial, termination of pregnancy is the best option to be discussed during parental counselling. ${ }^{4}$

There is paucity of literature as regards to knowledge and acceptability of prenatal diagnosis in the North Western part of the country and this informed the aim to conduct the study.

\section{Aims and objectives}

This was to determine the knowledge and acceptability of prenatal diagnosis among pregnant women receiving antenatal care at the Usmanu Danfodiyo University Teaching Hospital (UDUTH) Sokoto.

\section{METHODS}

This was a prospective cross sectional study among 417 pregnant women attending antenatal care clinic of the Usmanu Danfodiyo University Teaching Hospital (UDUTH) Sokoto. The questionnaire was developed based on literature search of similar studies on knowledge and acceptability of prenatal diagnosis., ${ }^{2,10,13-16}$ The questionnaire had 3 parts; the $1^{\text {st }}$ part consisted of sociodemographic characteristics of the participants, the $2^{\text {nd }}$ part assessed the knowledge of the participant on prenatal diagnosis. This assessed knowledge on who should have prenatal diagnosis, including the risk factors and various methods of conducting the diagnosis. While, the last part assessed general acceptability of prenatal diagnosis, method/s, and the result obtained and whether or not termination of pregnancy

The data collected was managed using the Statistical package for the Social Sciences (SPSS version 20. Frequency tables and graphs were generated for relevant variables. Descriptive statistics such as means and standard deviations were used to summarize quantitative variables while categorical variables were summarized with proportions. Knowledge score was generated from an aggregate of 15 items on knowledge related questions. Each correct answer was scored 1 and incorrect and unanswered questions were scored 0 . Thereby having a maximum score of " 15 " and a minimum score of" 0 ". The summation of each participant scores was used to calculate the overall knowledge score as follows; 0-5 poor knowledge, 6-10 some knowledge and 11-15 good knowledge. Comparison between knowledge score level and other variables was analysed with independent t-test.

\section{RESULTS}

Four hundred and seventeen women were recruited. Their ages ranged between 17 years and 45 years, with a mean age of $28.3 \pm 5.9$ years. The modal age group was 25-29 years- $123(30.4 \%)$. Majority of the respondents were Hausa/Fulani (55.2\%), Muslims (70\%), multigravida $(59.2 \%)$ and reside in the urban area $(84.7 \%)$. Over $4 / 5$ of them had some level of formal education. However, they were not gainfully employed. Table 1 summarized the socio-demographic characteristics.

The general knowledge on prenatal diagnosis was poor, as up to $340(81.5 \%)$ had little to no idea about the term prenatal diagnosis, women that should be screened and the 
various methods used. With only $18.5 \%$ having good knowledge. The educational status clearly affects the knowledge score of the respondent. The higher the level of education the better their understanding of prenatal diagnosis. Those group with tertiary level of education had some and good knowledge of prenatal diagnosis in about 28.8 and $6.1 \%$ respectively, compared to those with no formal education, primary and secondary level of education with a $\mathrm{p}<0.05$. There appears no statistical correlation between correspondent's occupation and that of their spouses with level of knowledge at $\mathrm{p}=0.23$ and 0.089 respectively.

Table 1: Socio-demographic characteristics of participants.

\begin{tabular}{|c|c|c|}
\hline Characteristic & Frequency & Percentage \\
\hline \multicolumn{3}{|l|}{ Age } \\
\hline $15-19$ & 30 & 72 \\
\hline $20-24$ & 90 & 216 \\
\hline $25-29$ & 123 & 29.5 \\
\hline $30-34$ & 100 & 24 \\
\hline$>35$ & 74 & 17.7 \\
\hline \multicolumn{3}{|l|}{ Parity } \\
\hline Primigravida & 78 & 18.7 \\
\hline Multiparea & 247 & 59.2 \\
\hline Grandmultiparae & 92 & 22.1 \\
\hline \multicolumn{3}{|l|}{ Tribe } \\
\hline Hausa/Fulani & 230 & 55.2 \\
\hline Yoruba & 52 & 12.3 \\
\hline Igbo & 72 & 17.3 \\
\hline Others & 63 & 15.2 \\
\hline \multicolumn{3}{|c|}{ Educational status } \\
\hline None & 68 & 16.3 \\
\hline Primary & 61 & 14.6 \\
\hline Secondary & 148 & 35.5 \\
\hline Tertiary & 140 & 33.6 \\
\hline \multicolumn{3}{|l|}{ Occupation } \\
\hline Unemployed & 185 & 44.4 \\
\hline Student & 66 & 15.8 \\
\hline Civil servant & 79 & 18.9 \\
\hline Self employed & 87 & 20.9 \\
\hline \multicolumn{3}{|c|}{ Spouse occupation } \\
\hline Unemployed & 40 & 9.6 \\
\hline Student & 43 & 10.3 \\
\hline Civil servant & 189 & 45.3 \\
\hline Self employed & 145 & 34.8 \\
\hline Total & 417 & 100 \\
\hline
\end{tabular}

The acceptability of prenatal diagnosis was generally good, as those who were willing to accept when offered accounted for $353(84.7 \%)$ while those who do not were $64(15.3 \%)$. Although, the reason for not accepting differs. Of the total that will not accept, $10(15.63 \%)$ believed was against their religion, 11 (17.19) fear the risk of abortion, $10(15.63 \%)$ thought the result may not be reliable and 33
$(51.56 \%)$ had no reason. The Figure 1 shows the reported acceptability of prenatal diagnosis.

There seems to be a correlation between the level of education and overall knowledge of prenatal diagnosis and its acceptability. However, parity and religion do not affect the correspondents' acceptability with a $\mathrm{p}$ value of 0.507 and 0.142 respectively.

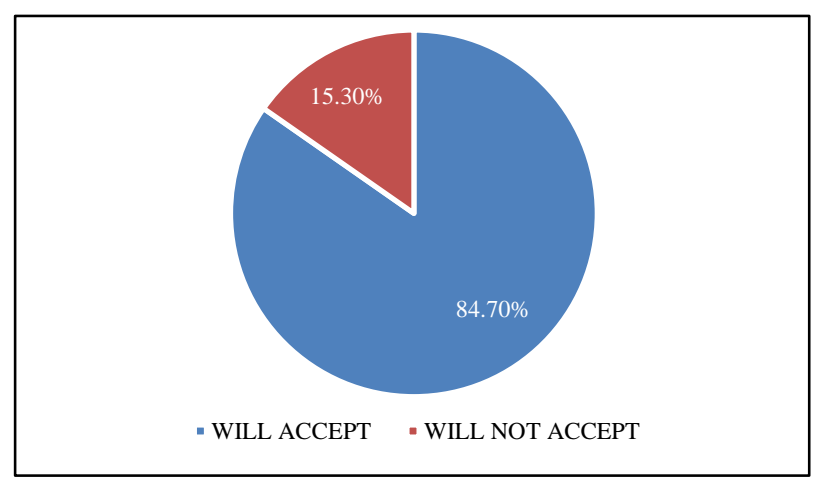

Figure 1: Acceptability of prenatal diagnosis.

Ultrasound appear to be the most preferred method of prenatal diagnosis by the respondent. This accounted for $332(94.05 \%)$ out of those that accepted to have prenatal diagnosis if indicated. Only $5(1.42 \%)$ will accept amniocentesis, $3(0.85 \%)$ chorionic villous samples, 26 $(7.37 \%)$ maternal serum for biochemistry and $2(0.57 \%)$ cordocentesis. This is summarized in Figure 2 below.

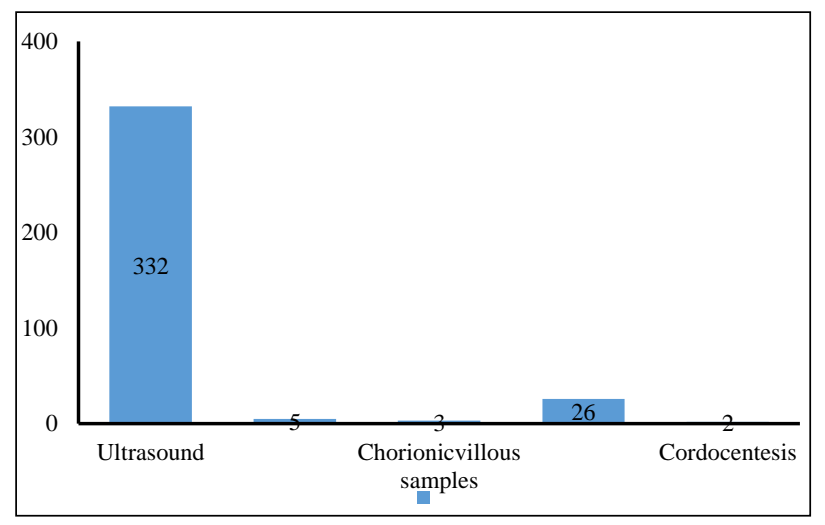

Figure 2: Frequency of preferred method/s of prenatal diagnosis.

All the correspondents that accepted to have prenatal diagnosis were willing to accept the result and possible treatment for the confirmed diagnosis.

Termination of pregnancy as an option of treatment in fetuses found to be having an abnormality as detected by prenatal diagnosis was only acceptable by 250 (70.82\%) respondents while, $167(47.31 \%)$ declined. Among those who declined, $77(46.11 \%)$ believed was against their religion, 29 (17.37\%) feared complications associated with termination of pregnancy, $27(43.11 \%)$ believed the result 
may not be reliable and $32(19.16 \%)$ had no particular reason.

There was statistical significant relationship between the respondents' educational status and overall knowledge of prenatal diagnosis as well as acceptability of termination of pregnancy, with a $p$ value of 0.04 and 0.02 respectively. However, their parity, religion and occupation did not affect their acceptance.

\section{DISCUSSION}

Prenatal diagnosis is a procedure that is yet to be popular in Nigeria, available data were mostly from advanced nations of the world. ${ }^{2}$

This study demonstrated that the overall knowledge of prenatal diagnosis is very low, as only $2.6 \%$ and $15.8 \%$ had good and some knowledge of prenatal diagnosis respectively, while $81.5 \%$ had poor to none. This is far lower than was obtained in similar studies in Ibadan; 55\% and Egypt; $44 \% .^{2,10}$ This may be as a result of better educational status attained by those respondent in the various study group. The respondent level of education is a major factor identified in this study group that determined the level of awareness of prenatal diagnosis. The higher their level of education, the more the understanding of prenatal diagnosis. This was the case in several other studies reported from Greece, Egypt and Ibadan. ${ }^{2,10,11}$ There seems to be no correlation between respondents' age, parity, religion and occupation with knowledge on prenatal diagnosis.

The acceptance of prenatal diagnosis in this study was high as about $84.7 \%$ will accept when indicated. This is in conformity with the findings from Cameroon that revealed $89.8 \%$, Egypt $88.8 \%$ and Pakistan $85.5 \% .^{10,13,14}$ It is however, higher than the result obtained from Ibadan; $68 \%$ and lower than that found in Greece; $95.2 \% .^{2,11}$ The level of educational and knowledge on prenatal diagnosis seem to influence the acceptability of prenatal diagnosis. As acceptability in those with secondary and tertiary level of education are $94.3 \%$ and $96.9 \%$ respectively. While those with primary and no formal education accounted for $84.8 \%$ and $77.2 \%$ respectively. All, $100 \%$ of those ever heard of prenatal diagnosis will accept it if offered. There seem to be no correlation between age, parity and religion on acceptance of prenatal diagnosis. This finding is similar to that found in a study from Greece. ${ }^{11}$ However, the acceptability of women in a study group in Ibadan, had only a weak correlation with knowledge of prenatal diagnosis, acceptance was more of attitudinal than awareness or knowledge. ${ }^{2}$ In another study, educational status significantly influences women's knowledge and their acceptance of prenatal screening but does not affect their acceptance of invasive procedures. ${ }^{10}$ Sixty-two percent of those that declined prenatal diagnosis had no particular reason, while $14.5 \%$ fear the risk of abortion, $12.5 \%$ believed it was against their religion and same proportion thought the result may not be reliable. The most widely method of choice was ultrasound in up to $86.2 \%$ of the respondents. This may not be unrelated to its awareness, availability, affordability, accessibility, noninvasiveness and risk free. The acceptability for maternal serum for biochemistry was $6.8 \%, 1.3 \%$ for amniocentesis, $0.8 \%$, chorionic villous sampling and $0.5 \%$, cordocentesis. This is in conformity with a study from Egypt, where $41 \%$ of respondent accepted invasive procedures, they however, declined to $31.6 \%$ later after having been informed of the risk of abortion. ${ }^{10}$

Termination of pregnancy as an option of management for affected fetus was accepted by majority of the respondent as about $250(63.1 \%)$ will accept in this study group. This is similar to $63 \%$ found in Pakistan and $62.5 \%$ in Cameroon. ${ }^{14,15}$ It was higher than that reported from north eastern Nigeria, $47 \% .{ }^{15}$ It is however lower than that seen in Egypt $72.6 \% .{ }^{10}$ Nigeria's abortion law makes it one of the most restrictive countries regarding abortion. ${ }^{17}$ This also would have made decision making regarding termination of pregnancy difficult.

In this study, only educational status and knowledge of prenatal diagnosis are the determinant of acceptance of termination of pregnancy. Other variables such as; age, parity, religion and occupation do not seem to influence the decision for termination of pregnancy. Similar result was obtained from Egypt. ${ }^{10}$ This was however not so in Cameroon where the determinant of acceptance for termination of pregnancy were single mothers and unemployment. ${ }^{13}$ In this study, among those that will not accept termination of pregnancy, $50.4 \%$ believed it was against their religion, $16.3 \%$ fear the complication of abortion, $15 \%$ thought the result may not be reliable while $18.4 \%$ had no reason for declining. However, religion has been a major factor militating against acceptability of prenatal diagnosis in North Eastern part of Nigeria. ${ }^{15}$

\section{CONCLUSION}

This study confirmed that there is overall poor knowledge on prenatal diagnosis. The level of education seems to be the single most important factor that determined the respondents' knowledge and acceptability of prenatal diagnosis including their decision for termination of pregnancy. Due to availability, affordability and safety of ultrasonography, almost the entire respondent will accept it as a diagnostic tool for prenatal investigation.

\section{Recommendations}

There is an obvious need for creating more awareness on the importance of prenatal diagnosis. This can be done during ANC, through media houses and channels and during community outreach. There should be a multisectorial policies that will enhance accessibility to health facilities and wider health insurance coverage. The Nigerian restrictive law for abortion should be reviewed to allow couples an option for termination of pregnancy in case of severe form of congenital anomaly. 


\section{ACKNOWLEDGMENTS}

We acknowledge the women who gave consent to participate in the study that may likely add to policy making regarding to improve patient care.

\section{Funding: No funding sources}

Conflict of interest: None declared

Ethical approval: The study was approved by the Institutional Ethics Committee

\section{REFERENCES}

1. Cunningham FG, Leveno KJ, Bloom SL, Hoffman BF, Spong YC, Dashe JS, et al. eds. Prenatal Diagnosis. In: Williams Obstetrics. 24th edn. New York; McGraw-Hill: 2014:283-297.

2. Adesina OAA, Oladapo OO, Adeniwu OF. The knowledge base and acceptability of prenatal diagnosis by pregnant women in Ibadan. Afr J Reprod Health. 2014;18(1):127-32.

3. Kwawukume EY, Arhin RA, Opoka BK. The Unborn Patient. In: Ekele BA, Danso KA, Emuveyan, eds. Comprehensive Obstetrics in the Tropics. 2nd edn. Accra Ghana: Assemblies of God literature center ltd.; 2015:34-40.

4. Rowan WC. Fetal medicine in clinical practice. In: Edmonds DK, ed. Dewhurst Textbook of Obstetrics and Gynaecology. 7th edition. Massachusetts, United States of America: Blackwell publishing; 2007:132143 .

5. Konar H, ed. DC Dutta's Textbook of Obstetrics, including perinatology and contraception. 7th edn. New Delhi: Jaypee Brothers medical Publishers Ltd; 2013:469-497.

6. NICE Clinical Guideline. Antenatal care: routine care for the healthy pregnant woman. The Organization for Economic Co-operation and Development (OECD). Family Database (Chart SF2.3. A). 2009.

7. Shobha HM, Roberts JS. Assessment of at-risk pregnancy. In: Decherney AH, Nathan N, Neri L, Roman AS, eds. Current diagnosis and treatment: OBSTETRICS and gynecology. 11th edn. New York: McGraw Hill; 2013:223-233.

8. Sarah V. Prenatal Diagnosis. In: Philip NB, Kenny LC, eds. Obstetrics by Ten Teachers. 19th edn. United Kingdom: Hodder and Stoughton; 2011:75-79.

9. Fadare JO. Some ethical issues in the prenatal diagnosis of sickle cell anaemia. Ann Ibadan Postgrad Med. 2009;7(2):26-8.
10. Rahaman HAS, Elhad RA, Gamal AM, Albadry A. Prenatal diagnosis in low resource setting: is it acceptable? J Obstet Gynaecol India. 2012;62(5):5159.

11. Karagkiouzis T, Sifakis S, Makrithanasis P, Dessypris N, Petridou ET, Kitsiou-Tzeli S, et al. Awareness of prenatal screening for fetal aneuploidy among pregnant women in Greece. In Vivo. 2015;29(1):15560.

12. Bricker L, Garcia J, Henderson J, Mugford M, Neilson $\mathrm{J}$, Roberts T, et al. Ultrasound screening in pregnancy: a systematic review of the clinical effectiveness, costeffectiveness and women's views. Database of Abstracts of Reviews of Effects (DARE): Qualityassessed Reviews. 2000.

13. Ambroise N, Alfred KN, Dora M, Jeanne N, Caryl Z. Acceptability of prenatal diagnosis by sample of parents of sickle cell anaemia patient in Cameroon (Sub-Saharan African). J Genet Couns. 2011;20:47685.

14. Arif MO, Fatmi Z, Pardeep B, Ali T, Iqbal H, Bangash $\mathrm{HK}$, et al. Attitudes and perceptions about prenatal diagnosis and induced abortion among adults of Pakistani population. Prenat Diagn. 2008;28(12):1149-55.

15. Kagu MB, Abja UA, Ahmed SG. Awareness and acceptability of prenatal diagnosis of sickle cell anaemia among health professionals and students in North Eastern Nigeria. Niger J Med. 2004;13(1):4851.

16. Ajah OL, Nwali SA, Amah CC, Nwankwo TO, Lawani LO, Ozumba BC. Attitude of reproductive health care providers to prenatal diagnosis in a low resource Nigerian setting. J Diag Res. 2017;11(2):4-7.

17. Panel Code (Northern States) Federal Provision Act, Chapter 345 of the laws of the Federation of Nigeria (Revised ed. 1990), Articles 232-236. Nigeria's Abortion Provisions. Center for Reproductive Rights. Available from: https://maps.reproductiverights.org/ world-abortion-laws/nigerias-abortion-provisions. Accessed on 2 October 2017.

Cite this article as: Ahmed Y, Panti AA, Umar AG, Funtua AR, Abdullahi N, Garba JA. Knowledge and acceptability of prenatal diagnosis among pregnant women attending antenatal clinic in a tertiary health institution in Sokoto, Nigeria. Int J Reprod Contracept Obstet Gynecol 2021;10:3678-83. 